\title{
Impact of intraoperative radiotherapy on the perioperative period of patients after breast-conserving surgery
}

\author{
Xiaowu Hu${ }^{1}$, Yong $\mathrm{He}^{2}$, Xinxin Chen ${ }^{1}$, Ting Xia ${ }^{1}$, Tengfei Cao ${ }^{1}$, Haixia Jia ${ }^{1}$, Lehong Zhang $^{1}$ \\ ${ }^{1}$ Department of Breast Surgery, the Second Affiliated Hospital of Guangzhou Medical University, Guangzhou, China; ${ }^{2}$ Department of Radiotherapy, \\ the Second Affiliated Hospital of Guangzhou Medical University, Guangzhou, China \\ Contributions: (I) Conception and design: L Zhang, X Hu; (II) Administrative support: Y He; (III) Provision of study materials or patients: T Xia; \\ (IV) Collection and assembly of data: T Cao, H Jia; (V) Data analysis and interpretation: X Chen; (VI) Manuscript writing: All authors; (VII) Final \\ approval of manuscript: All authors. \\ Correspondence to: Lehong Zhang. Department of Breast Surgery, the Second Affiliated Hospital of Guangzhou Medical University, Guangzhou \\ 510260, China. Email: rxwk3390@sina.com.
}

\begin{abstract}
Background: To investigate the effect of intraoperative radiotherapy (IORT) in the perioperative period of patients after breast-conserving surgery (BCS).

Methods: The clinical data of 100 patients with early breast cancer undergoing breast-conserving surgery (BCS) followed by treatment with IORT using the Intrabeam system (Carl Zeiss Meditec, Oberkochen, Germany) (BCS + IORT group, n=100) between June 2016 and December 2019 were analyzed and compared with the data of 60 matched patients who only underwent breast-conserving therapy over the same period (BCS group, $\mathrm{n}=60$ ). The surgical settings and postoperative acute complications between the groups were assessed.
\end{abstract}

Results: There was no significant statistical difference between the groups in terms of age, tumor size, grading, lymph node status, hormone receptor status, and human epidermal growth factor receptor 2 (HER-2) status $(\mathrm{P}>0.05)$. The BCS + IORT group had a significantly longer surgery duration $(\mathrm{P}<0.05)$, but there was no significant statistical difference in terms of intraoperative blood loss, amount of bleeding, drainage tube removal time, postoperative length of hospitalization, incision suture removal time, or incidence of postoperative complications $(\mathrm{P}>0.05)$.

Conclusions: IORT using the Intrabeam system safely delivers radiation therapy, is well-tolerated, has acceptable acute toxicity, and does not significant increase the risk of surgery or the incidence of perioperative complications.

Keywords: Early breast cancer; breast-conserving surgery (BCS); intraoperative radiotherapy; Intrabeam system

Submitted Sep 01, 2020. Accepted for publication Oct 12, 2020.

doi: $10.21037 /$ gs-20-727

View this article at: http://dx.doi.org/10.21037/gs-20-727

\section{Introduction}

Breast cancer (BC) is one of the most common malignant neoplasms in women, and treatment with total mastectomy may adversely affect the patient's psychology and aesthetic satisfaction. The combination of breastconserving surgery therapy (BCS) and whole breast radiation therapy (WBRT) has become one of the major treatment paradigms of early BC. A number of studies have confirmed that breast conserving surgery combined with adjuvant radiotherapy has the same effect as mastectomy. Postoperative whole breast radiotherapy is an important clinical treatment for early breast cancer, which can effectively improve the quality of life of patients $(1,2)$. Although postoperative adjuvant radiotherapy has been proved to be beneficial, there are still some patients who did not receive radiotherapy after tumor resection due to long postoperative radiotherapy cycle, cumbersome travel 
to hospital, and worry about the side effects of multiple radiotherapy (3). Secondly, conventional radiotherapy is usually started 4-6 months after surgery, which affects the accuracy of tumor bed location; moreover, the $\alpha / \beta$ ratio of breast tissue is low, so conventional fractionation radiotherapy may not achieve the best radiobiological effect. In addition, patients with traditional fractionated extracorporeal radiotherapy must bear the dual burden of body and mind (4): rough breast skin, deepening pigmentation and atrophy of local skin or prosthesis. It not only seriously affects the beauty of breast, but also reduces the quality of life of patients. Intraoperative radiotherapy (IORT), including accelerated partial breast irradiation (APBI) and targeted intraoperative radiotherapy (TARGIT-IORT), delivers all radiation directly to the tumor bed during lumpectomy, allowing all radiation to be delivered in a single dose to the region where recurrence would most likely occur. This method has the additional merits of being able to accurately position the target region under direct vision during operation, protecting surrounding normal tissue, and shortening the course of treatment (5). For some patients with selective early $\mathrm{BC}$, IORT can even confer satisfactory local control rate and good cosmetic effect (6). As the largest related international prospective randomized phase III clinical study, the TARGIT-A study $(7,8)$ showed that IORT can replace external radiation therapy for the treatment of patients with selective breast cancer in the early stage. Meanwhile, the Mannheim Medical Center reported $(9,10)$ that for early selective breast cancer, a $50 \mathrm{kv} \mathrm{X}$-ray is safe and effective. Although these results indicate that the effect of intraoperative radiotherapy is significant, intraoperative radiotherapy has not been widely used in clinical practice, and it is still not one of the standard treatment options for breast conserving surgery of early breast cancer. Therefore, it is still necessary to further study the clinical and anticancer mechanism of intraoperative radiotherapy for breast cancer. Indeed, considerable research has focused on the oncologic safety, acute/chronic toxicity, and cosmetic effect of BC treatment, but few studies have evaluated the perioperative application of IORT in depth. We thus conducted a retrospective analysis of the data of 160 early BC patients collected from June 2016 to December 2019, the results of which are detailed in this paper. We present the following article in accordance with the STROBE reporting checklist (available at http:// dx.doi.org/10.21037/gs-20-727).

\section{Methods}

\section{General data}

A retrospective analysis of 160 early BC patients who underwent BCS from the period between June 2016 and December 2019 in the Department of Breast Surgery at the Second Affiliated Hospital of Guangzhou Medical University was conducted. Of these patients, 100 (62.5\%) were women who underwent IORT during BCS, with IORT being applied using the Intrabeam system. The study protocol was approved by the hospital ethics committee (No.2016-hs-10). Mammography, sonography, and magnetic resonance imaging (MRI) were used in all patients before surgery. The inclusion criteria were as follows: (I) desire for breast-conserving surgery, (II) unifocal tumor size $\leq 3 \mathrm{~cm}$, (III) age $>35$ years, (IV) negative margins after resection, (V) clinical and radiologic N0 patients. The exclusion criteria were as follows: (I) multifocal patients with extensive intraductal component, (II) previous history of radiotherapy on the ipsilateral breast, (III) patients with bilateral BC, (IV) inflammatory breast cancer. The Breast surgeon communicate with patients and their families to inform them of the risks and meanings of IORT. Patients were enrolled in either the BCS + IORT group or the BCS group in accordance with their opinion. All procedures performed in this study involving human participants were in accordance with the Declaration of Helsinki (as revised in 2013). Individual consent for this retrospective analysis was waived.

\section{Surgery}

\section{Breast-conserving surgery}

First, clinically negative axillary lymph nodes were subjected to biopsy by methylene blue dying for sentinel nodes. Generally, it was required that at least 3 sentinel nodes be excised. If the frozen biopsy yielded a positive result, the axillary lymph nodes would be directly dissected; otherwise, the axillary lymph nodes would be exempt from dissection. Next, the tumor mass was excised under general anesthesia according to palpation or localization results to 1-2 cm beyond the surrounding normal tissues; tissues were sampled intraoperatively at 10 points surrounding the tumor cavity and at the basilar part and subjected to frozen biopsy. Finally, if the result was positive, the excision would be extended by $1 \mathrm{~cm}$ (for up to 2 times); otherwise, the tumor bed would be subjected to IORT with Intrabeam system 
(20 Gy to the surface of the applicator) with an adapter of appropriate size $(2-5 \mathrm{~cm})$. Upon the completion of the radiotherapy, the tumor cavity was fixed with 4 titanium clamps. After an extensive surgery, the surrounding flap tissue was widely released, and negative pressure drainage was performed and sutured in stage I. Upon completion of the surgery, for patients in the BCS group who were exempt from IORT.

\section{Radiotherapy}

\section{Intraoperative radiotherapy}

If the frozen biopsy of the residual cavity yielded a negative result after the extended resection of the tumor, the size of residual cavity was measured and reported to the radiation oncologist, and IORT was delivered using the Intrabeam system with a $2.0-5 \mathrm{~cm}$ adapter. A dose of 20 Gy was delivered to the surface of the spherical applicator for IORT for a median duration of 25 minutes (range, 1247 minutes). The edges of the skin incision were everted so any part of skin was at least $1 \mathrm{~cm}$ away from the applicator surface and protected with moist aseptic gauze to avoid excessive radiation exposure. Conventional whole breast external beam radiotherapy (EBRT) was administered (50 Gy in 25 fractions, tumor bed boost omitted) postoperatively to patients with high-risk factors (metastatic axillary lymph nodes $\geq \mathrm{R} 3$, aged $\leq 60$, lymphatic vessel invasion/lymphovascular invasion, invasive lobular carcinoma, etc.)

\section{Subsequent therapy}

Subsequent chemotherapy, endocrine therapy, and targeted therapy regimens of all patients were carried out in accordance with National Comprehensive Cancer Network guidelines (NCCN) guidelines and conventional whole breast EBRT was delivered 2 weeks after the completion of chemotherapy (or after the wounds healed).

\section{Statistical analysis}

SPSS 26.0 was used for data analysis. Measurement data in normal distribution are expressed as mean \pm standard deviation $(\bar{x} \pm \mathrm{s})$; a $t$-test was carried out for intergroup comparisons of two sets of independent, normally distributed data with a homogeneity of variance; enumeration data are provided either in the number of cases or percentages and were subjected to chi square test or Fisher's exact probability test. A P value $<0.05$ was considered statistically significant.

\section{Results}

The analysis of general clinical patient data from June 2016 to December 2019 revealed that 160 female breast cancer patients underwent BCS at the Second Affiliated Hospital of Guangzhou Medical University. There was no difference of statistical significance $(\mathrm{P}>0.05)$ between the BCS group + IORT group and the BCS group in terms of age, tumor size, oncology grading, hormone receptor status, and human epidermal growth factor receptor 2 (HER-2) status (Table 1).

\section{Comparison of intraoperative conditions}

There was no significant difference between the groups in terms of amount of bleeding, length of postoperative hospitalization, and time to extubation; however, the surgery duration in the BCS + IORT group was significantly prolonged, and the difference was statistically significant $(\mathrm{P}<0.05)$ (Table 2).

\section{Comparison of postoperative complications}

There were no significant statistical differences $(\mathrm{P}>0.05)$ between the groups in terms of the incidences of seroma, hematoma, infection, or erythema (Table 3).

\section{Discussion}

In 1889, Stephen Paget, a British surgeon, found that the distribution of metastatic cancer was not random. Tumor cells had a special growth tendency to some organs and environments. He was the first to put forward the "seed soil" theory. In this hypothesis, he compares cancer cells to "seeds" and the destination of cancer metastasis to "soil". In other words, cancer cells can spread around like seeds, but seeds only grow in fertile soil. Therefore, it is considered that tumor metastasis is a special tumor cell (seed), which can survive in a suitable microenvironment (soil) of tumor bed (11). Surgery may change the microenvironment of tumor bed, stimulate the proliferation of residual tumor cells, and increase the risk of local recurrence near the primary tumor after breast conserving surgery. The drainage fluid produced by breast conserving surgery and intraoperative radiotherapy 
Table 1 Comparison of the general clinical data between the BCS + IORT group and the BCS group

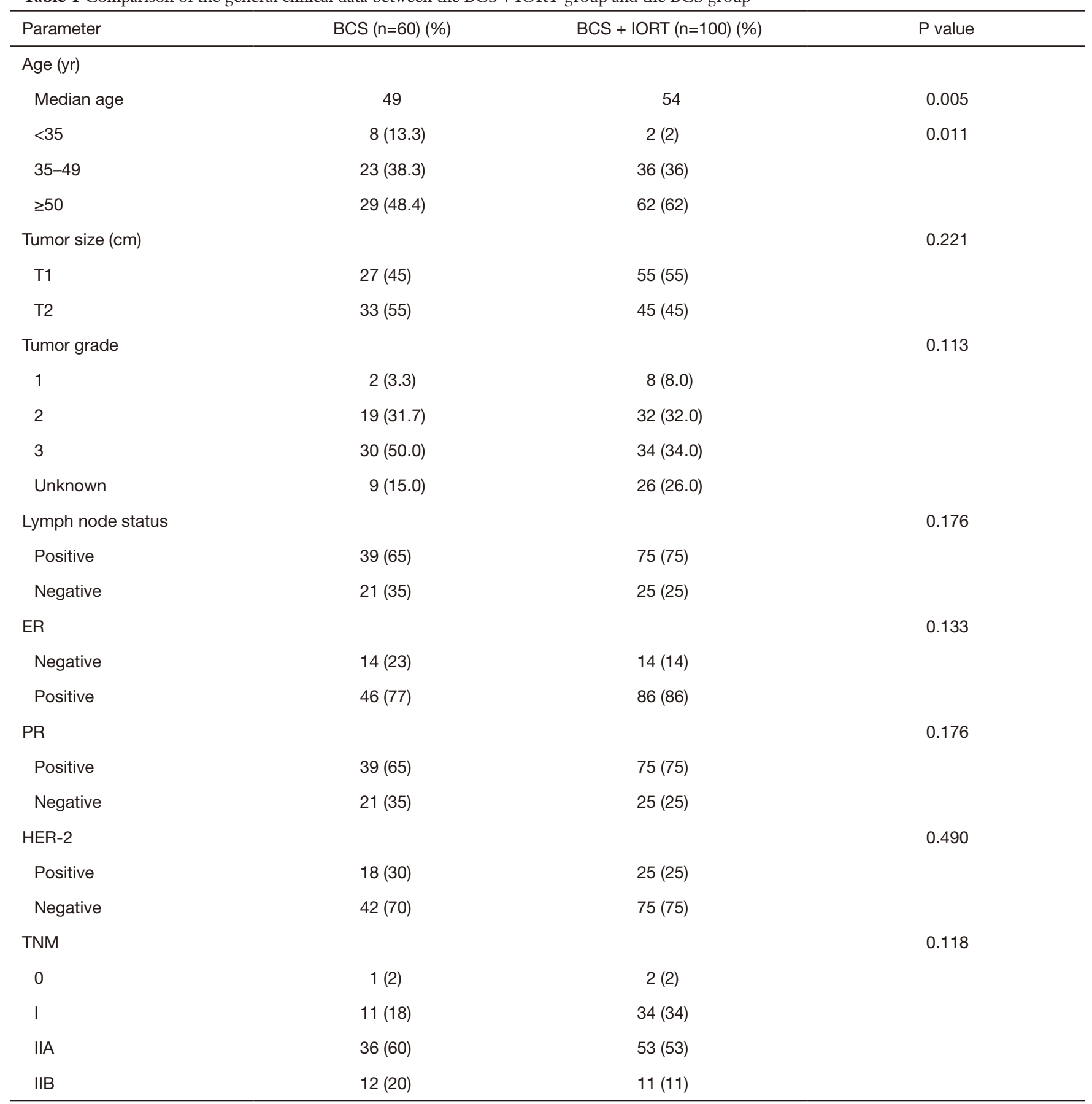

BCS, breast-conserving surgery; IORT, intraoperative radiotherapy; ER, estrogen receptor; HER-2, human epidermal growth factor receptor 2; PR, progesterone receptor; TMN, tumor-node-metastasis.

"soaks" the "soil", resulting in increased expression of anti-tumor cytokines such as IL-4, IL-5, IL-13, TNF and IFN. At the same time, the expression of cytokines positively related to tumor proliferation, migration and invasion is decreased, such as VEGF-R, gro-1, etc. (12). A single high-dose radiation during operation is more conducive to destroying tumor microvessels (13), improving the recognition and killing effect of immune cells in 
Table 2 Comparison of intraoperative and postoperative conditions between the BCS + IORT group and the BCS group

\begin{tabular}{lcccc}
\hline Parameter & BCS $(\mathrm{n}=60)$ & BCS + IORT $(\mathrm{n}=100)$ & $t$ value & P value \\
\hline Surgery duration (min) & $159.3 \pm 36.7$ & $207.5 \pm 36.2$ & -5.82 & -1.73 \\
Blood loss $(\mathrm{mL})$ & $37.3 \pm 10.1$ & $40.1 \pm 10.2$ & -2.60 & 0.08 \\
Drainage tube removal time (d) & $5.0 \pm 1.6$ & $5.9 \pm 1.2$ & 0.26 & 0.05 \\
Postoperative hospital stay & $12.4 \pm 3.7$ & $12.6 \pm 3.2$ & -0.97 & 0.37 \\
Incision suture removal time & $12.7 \pm 1.9$ & $13.0 \pm 2.5$ & 0.34 \\
\hline
\end{tabular}

*, significant difference. BCS, breast-conserving surgery; IORT, intraoperative radiotherapy.

Table 3 Incidences of postoperative complications in the BCS + IORT group and the BCS group

\begin{tabular}{|c|c|c|c|}
\hline Parameter & $\operatorname{BCS}(n=60)$ & BCS + IORT $(n=100)$ & $P$ value \\
\hline Seroma needing more than three aspirations & $2(3.4 \%)$ & $6(6.0 \%)$ & 0.708 \\
\hline Infection needing intravenous antibiotics or surgical intervention & $2(3.4 \%)$ & $5(5.0 \%)$ & 0.921 \\
\hline Skin breakdown or delayed wound healing & $2(3.4 \%)$ & $7(7.0 \%)$ & 0.535 \\
\hline
\end{tabular}

BCS, breast-conserving surgery; IORT, intraoperative radiotherapy.

tumor microenvironment, such as CD8 + T cells (14), resulting in changes in tumor microenvironment And free radical attack biological macromolecules (nucleic acid, protein, enzyme and ester) lead to biomolecule damage, which is more effective than conventional radiotherapy in killing breast cancer stem cells (15), and affecting multiple cell pathways (16-18) and interfering with DNA damage repair $(19,20)$, so as to achieve the effect of destroying tumor cells. The technological bottleneck of IORT has recently been overcome as a result of the emergence of compact linear accelerators, which are free from the drawbacks of conventional radiotherapy equipment, such as the need for special shielding protection, immobility, long anesthesia duration, and conveyance of the patient during therapy $(21,22)$. The Intrabeam system has been widely used in clinical practice due to its low-energy $(50 \mathrm{kV})$ $\mathrm{X}$-ray, low penetrability, fast attenuation of radiation dose, and a lack of special shielding protection requirements. In a comparative study by Vaidya et al. (23), the 5-year recurrence-free survival rates in the TARGIT group and ERBT group were $93.9 \%$ and $92.5 \%$, respectively. The difference between the groups was not statistically significant $(\mathrm{P}=0.35)$, demonstrating that the delivery of IORT using the Intrabeam system is safe, feasible, and effective. Many of the relevant studies have mainly focused on the safety (24), acute/chronic toxicity, and cosmetic effect of BC treatment $(25,26)$, while little focus has been placed on the influence of acute toxicity on the perioperative application of IORT. The primary objective of our current study was thus to analyze the perioperative effect of IORT with Intrabeam system on early BC patients who underwent BCS. In the international, multicenter, prospective randomized control TARGIT-A study $(25,26), 2,232$ early BC patients aged above 45 were recruited with 1,113 of them being randomized into the IORT group, in which the incidence of seroma was $3.1 \%$ and the wound infection rate was $1.8 \%$. In our study, the incidence of seroma was $6.0 \%$ and the infection rate was $5.0 \%$; the infection rate was thus slightly higher than that in the TARGIT-A study (Table 4), but was comparable to the matched patients undergoing BCS over the same period. The wound infection rate arising from IORT varies significantly in the currently available studies, ranging from $2.3 \%$ to $13.6 \%(27-29)$. This difference might be attributable to the different radiotherapy equipment and methods used in the studies, and to the effect that variable patient characteristics, surgical procedures, and postoperative therapies might have had on toxicity and side effects. It was reported (30) that factors like old age, obesity, diabetic anemia, chronic obstructive pulmonary disease, large tumor size, and smoking could have adverse influence on the complications in operative wounds of BC surgery. This is evident from the distribution 
Table 4 Incidences of postoperative complications in the TARGIT-A group and BCS + IORT group

\begin{tabular}{llr}
\hline Parameter & TARGIT-A & BCS + IORT $(\mathrm{n}=100)$ \\
\hline Hematoma needing surgical evacuation & $11(1.0 \%)$ & $1(1.0 \%)$ \\
Seroma needing more than three aspirations & $23(2.1 \%)$ & $6(6.0 \%)$ \\
Infection needing intravenous antibiotics or surgical intervention & $20(1.8 \%)$ & $5(5.0 \%)$ \\
Skin breakdown or delayed wound healing & $31(2.8 \%)$ & $7(7.0 \%)$ \\
\hline
\end{tabular}

BCS, breast-conserving surgery; IORT, intraoperative radiotherapy.

characteristics of patients who experienced infection in their operative incision: 1 patient had a large tumor excised and subjected to RT with a $5 \mathrm{~cm}$ adapter for as long as 45 minutes; 3 patients were 70 years old; 1 patient had a BMI of 35.5 (probably an important factor that contributed to the infection); indeed, Ahn et al. (31) identified advanced age and high body mass index (BMI) as risk factors for wound complications. The use of prophylactic antibiotics can reduce the incidence of wound infection. Of the 5 patients who experienced wound infection, only 1 had been administered prophylactic antibiotics. Of the 5 patients with wound infection in the IORT group, 3 had been identified with Staphylococcus aureus infection. These patients' incision was healed later after having been opened up, fully drained, and treated with antibiotics; 2 had not been identified with any bacterial infection, and their incision was healed after having been opened up and fully drained; the median time to the recovery of these patients was 4.0 (range, 1-11) months. One patient in the BCS group experienced incision infection, and culture test revealed $S$. aureus infection; the patient received anti-infection treatment, and the wound was healed 3 weeks later. The healing performance of patients in the IORT group was significantly decreased, and this decrease might be associated with the blood vessel damage and tissue fibrosis caused by a large dose of irradiation.

In our study, the operative duration of BCS was $159.3 \pm 36.7$ minutes, which is a bit higher than the duration of $128.3 \pm 31.2$ minutes reported in the reference literature (32). This could be related to the number of frozen tissue samples taken during the surgery. It was a routine practice in our study that samples be taken at 1-10 sites in the residual cavity for intraoperative frozen pathological examination. This generally took 1 hour, and consequently the surgery duration was prolonged. The surgery duration in the BCS group was significantly shorter than that in the IORT group, probably because the preparation of radiation equipment, double purse-string suturing, wrapping of the adapter, and the delivery of IORT are time-consuming. Finally, there was no statistical difference between the groups in terms of intraoperative bleeding and time to extubation.

In conclusion, the delivery of IORT merely prolongs the operation time without an accompanying rise in the incidence of perioperative complications. IORT using the Intrabeam system can safely deliver radiation therapy, is well-tolerated, has acceptable acute toxicity, and does not increase the risk of surgery or the incidence of perioperative complications.

\section{Acknowledgments}

Funding: Funding was provided by the Clinical Study and Application of New Technology and New Business, the Second Affiliated Hospital of Guangzhou Medical University (no. 2017-XJS-Y-02).

\section{Footnote}

Reporting Checklist: The authors have completed the STROBE reporting checklist. Available at http://dx.doi. org/10.21037/gs-20-727

Data Sharing Statement: Available at http://dx.doi. org/10.21037/gs-20-727

Conflicts of Interest: All authors have completed the ICMJE uniform disclosure form (available at http://dx.doi. org/10.21037/gs-20-727). The authors have no conflicts of interest to declare.

Ethical Statement: The authors are accountable for all aspects of the work in ensuring that questions related to the accuracy or integrity of any part of the work are appropriately investigated and resolved. All procedures performed in this study involving human participants were 
in accordance with the Declaration of Helsinki (as revised in 2013). Individual consent for this retrospective analysis was waived. The study protocol was approved by the hospital ethics committee (No.2016-hs-10).

Open Access Statement: This is an Open Access article distributed in accordance with the Creative Commons Attribution-NonCommercial-NoDerivs 4.0 International License (CC BY-NC-ND 4.0), which permits the noncommercial replication and distribution of the article with the strict proviso that no changes or edits are made and the original work is properly cited (including links to both the formal publication through the relevant DOI and the license). See: https://creativecommons.org/licenses/by-nc-nd/4.0/.

\section{References}

1. Fisher B, Anderson S, Bryant J, et al. Twenty-year followup of a randomized trial comparing total mastectomy, lumpectomy, and lumpectomy plus irradiation for the treatment of invasive breast cancer. $\mathrm{N}$ Engl J Med 2002;347:1233-41.

2. Odom EB, Mehta N, Parikh RP, et al. Paravertebral Blocks Reduce Narcotic Use Without Affecting Perfusion in Patients Undergoing Autologous Breast Reconstruction. Ann Surg Oncol 2017;24:3180-7.

3. LeMasters TJ, Madhavan SS, Sambamoorthi U, et al. Disparities in the Initial Local Treatment of Older Women with Early-Stage Breast Cancer: A Population-Based Study. J Womens Health (Larchmt) 2017;26:735-44.

4. Gao Y, Li X, Zhong H, et al. Dosimetric Comparison Between Prone and Supine Positions in Radiotherapy on Organs at Risk after Breast-conserving Surgery: A Metaanalysis. Cancer Res Prev Treat 2015;42:905-10.

5. Herskind C, Steil V, Kraus-Tiefenbacher U, et al. Radiobiological aspects of intraoperative radiotherapy (IORT) with isotropic low-energy $\mathrm{X}$ rays for early-stage breast cancer. Radiat Res 2005;163:208-15.

6. Njeh CF, Saunders MW, Langton CM. Accelerated Partial Breast Irradiation (APBI): A review of available techniques. Radiat Oncol 2010;5:90.

7. Vaidya JS, Joseph DJ, Tobias JS, et al. Targeted intraoperative radiotherapy versus whole breast radiotherapy for breast cancer (TARGIT-A trial): an international, prospective, randomised, non-inferiority phase 3 trial. Lancet 2010;376:91-102.

8. Welzel G, Boch A, Sperk E, et al. Radiation-related quality of life parameters after targeted intraoperative radiotherapy versus whole breast radiotherapy in patients with breast cancer: results from the randomized phase III trial TARGIT-A. Radiat Oncol 2013;8:9.

9. Veronesi U, Orecchia R, Luini A, et al. Intraoperative radiotherapy during breast conserving surgery: a study on 1,822 cases treated with electrons. Breast Cancer Res Treat 2010;124:141-51.

10. Sperk E, Welzel G, Keller A, et al. Late radiation toxicity after intraoperative radiotherapy (IORT) for breast cancer: results from the randomized phase III trial TARGIT A. Breast Cancer Res Treat 2012;135:253-60.

11. Paget $\mathrm{S}$. The distribution of secondary growths in cancer of the breast. 1889. Cancer Metastasis Rev 1989;8:98-101.

12. Belletti B, Vaidya JS, D'Andrea S, et al. Targeted intraoperative radiotherapy impairs the stimulation of breast cancer cell proliferation and invasion caused by surgical wounding. Clin Cancer Res 2008;14:1325-32.

13. Garcia-Barros M, Paris F, Cordon-Cardo C, et al. Tumor response to radiotherapy regulated by endothelial cell apoptosis. Science 2003;300:1155-9.

14. Lee Y, Auh SL, Wang Y, et al. Therapeutic effects of ablative radiation on local tumor require CD8+ $\mathrm{T}$ cells: changing strategies for cancer treatment. Blood 2009;114:589-95.

15. Phillips TM, McBride WH, Pajonk F. The response of CD24(-/low)/CD44+ breast cancer-initiating cells to radiation. J Natl Cancer Inst 2006;98:1777-85.

16. Huang $W$, Zhang $H$, Jiang X, et al. Effects of hedge signaling pathway on proliferation, apoptosis and cyclinD1 expression of breast cancer cell line MDA-MB-231. Journal of Chongqing Medical University 2009;34:45-8.

17. Dong Y, Sun Y, Huang Y, et al. Depletion of MLKL inhibits invasion of radioresistant nasopharyngeal carcinoma cells by suppressing epithelial-mesenchymal transition. Ann Transl Med 2019;7:741.

18. Phillips TM, McBride WH, Pajonk F. The response of CD24(-/low)/CD44+ breast cancer-initiating cells to radiation. J Natl Cancer Inst 2006;98:1777-85.

19. Kadouri L, Kote-Jarai Z, Hubert A, et al. A singlenucleotide polymorphism in the RAD51 gene modifies breast cancer risk in BRCA2 carriers, but not in BRCA1 carriers or noncarriers. Br J Cancer 2004;90:2002-5.

20. Uegaki K, Adachi N, So S, et al. Heterozygous inactivation of human $\mathrm{Ku} 70 / \mathrm{Ku} 86$ heterodimer does not affect cell growth, double-strand break repair, or genome integrity. DNA Repair (Amst) 2006;5:303-11.

21. Schneider F, Fuchs H, Lorenz F, et al. A novel device for intravaginal electronic brachytherapy. Int J Radiat Oncol 
Biol Phys 2009;74:1298-305.

22. Eaton DJ, Gonzalez R, Duck S, et al. Radiation protection for an intra-operative X-ray device. Br J Radiol 2011;84:1034-9.

23. Vaidya JS, Wenz F, Bulsara M, et al. An international randomised controlled trial to compare TARGeted Intraoperative radioTherapy (TARGIT) with conventional postoperative radiotherapy after breast-conserving surgery for women with early-stage breast cancer (the TARGIT-A trial). Health Technol Assess 2016;20:1-188.

24. Lai HW, Liu LC, Ouyang F, et al. Multi-center study on patient selection for and the oncologic safety of intraoperative radiotherapy (IORT) with the Xoft Axxent ${ }^{\circledR}$ $\mathrm{eBx} ®$ System for the management of early stage breast cancer in Taiwan. PLoS One 2017;12:e0185876.

25. Kraus-Tiefenbacher U, Bauer L, Kehrer T, et al. Intraoperative radiotherapy (IORT) as a boost in patients with early-stage breast cancer -- acute toxicity. Onkologie 2006;29:77-82.

26. Cracco S, Semprini G, Cattin F, et al. Impact of intraoperative radiotherapy on cosmetic outcome and complications after oncoplastic breast surgery. Breast J 2015;21:285-90.

Cite this article as: $\mathrm{Hu} \mathrm{X}, \mathrm{He} \mathrm{Y,} \mathrm{Chen} \mathrm{X,} \mathrm{Xia} \mathrm{T,} \mathrm{Cao} \mathrm{T,} \mathrm{Jia} \mathrm{H,}$ Zhang L. Impact of intraoperative radiotherapy on the perioperative period of patients after breast-conserving surgery. Gland Surg 2020;9(5):1535-1542. doi: 10.21037/gs-20-727
27. d'Illiers LB, Lafay F, Faure C, et al. Up to 5 years outcomes following intraoperative radiotherapy for early breast cancer. Transl Cancer Res 2018;7:859-70.

28. Wersal C, Keller A, Weiss C, et al. Long-term changes in blood counts after intraoperative radiotherapy for breast cancer-single center experience and review of the literature. Transl Cancer Res 2019;8:1882-903.

29. Jacobs DHM, Speijer G, Petoukhova AL, et al. Acute toxicity of intraoperative radiotherapy and external beamaccelerated partial breast irradiation in elderly breast cancer patients. Breast Cancer Res Treat 2018;169:549-59.

30. El-Tamer MB, Ward BM, Schifftner T, et al. Morbidity and mortality following breast cancer surgery in women: national benchmarks for standards of care. Ann Surg 2007;245:665-71.

31. Ahn SG, Bae SJ, Lee HW, et al. A phase II study investigating the acute toxicity of targeted intraoperative radiotherapy as tumor-bed boost plus whole breast irradiation after breast-conserving surgery in Korean patients. Breast Cancer Res Treat 2019;174:157-63.

32. Fan P, Lv P, Li J, et al. Analysis of clinical efficacy of breast cancer and improved radical surgery. Chinese Journal of Cancer Prevention and Treatment 2018;25:52-4. 